\title{
Effect of hydrophobic group on flocculation properties and dewatering efficiency of cationic acrylamide copolymers
}

\author{
Haijing Ren ${ }^{\mathrm{a}}$, Wenna Chen ${ }^{\mathrm{b}}$, Yunfei Zheng ${ }^{\mathrm{b}}$, Zhaokun Luan ${ }^{\mathrm{a}, *}$ \\ ${ }^{a}$ State Key Laboratory of Environmental Aquatic Chemistry, Research Center for Eco-Environmental Sciences, \\ Chinese Academy of Sciences, Beijing 100085, China \\ b Department of Chemistry, Guangxi Teachers' College, Nanning, Guangxi 530001, China
}

Received 28 May 2006; received in revised form 17 December 2006; accepted 30 March 2007

Available online 14 April 2007

\begin{abstract}
Three kinds of hydrophobically modified copolymers of acrylamide with 3-acrylamido-2-hydroxypropyltrialkylammonium chloride were prepared, in which alkyls were ethyl, butyl and octyl, respectively. The conformations of the hydrophobically modified copolymers were studied using TEM micrograph. The flocculation performances and the dewatering efficiencies were investigated. The results showed that the hydrophobic group could enhance the flocculation properties. The longer the hydrophobic alkyl chain was, the better the flocculation result was. Compared with poly-diallyldimethyl ammonium chloride (PDADMAC) and poly-acrylamide (PAM), the hydrophobically modified polymers showed better performances in turbidity removal and were quite more efficient in dewatering examination.
\end{abstract}

(c) 2007 Published by Elsevier Ltd.

Keywords: Hydrophobic group; Copolymer; Flocculation; Dewater; TEM

\section{Introduction}

Flocculation is one of the most important processes in wastewater treatment to depurate water removing dissolved, colloidal and suspended particles. In this process, flocculant is undoubtedly the most important factor. In recent years, great efforts have been made to study water-soluble polymers, which can be used as flocculant. Research of polyacrylamide and its copolymers is one of the most popular hot aspects because the reasonable cost,

\footnotetext{
${ }^{*}$ Corresponding author. Tel./fax: +86 1062849198.

E-mail addresses: haijing79@gmail.com (H. Ren), luanzk@ mail.rcees.ac.cn (Z. Luan).
}

easy polymerization and good-treatment result. Different kinds of derivates of poly-acrylamide have been prepared in order to treat different kinds of wastewater, and improved flocculation performances were demonstrated $[1-4]$. In the past few decades, researchers have found hydrophobically modified polymers showed excellent properties in the oily wastewater treatment in oil recovery operations and were effective in cleaning up organic contaminants in wastewater. These polymers include hydrophobically associating polyacrylate [5,6], vinyl trimethyloxysilane (VTMS) modified PDADMAC [7], hydrophobically modified chitosan [8], hydrophobically modified polycations [9], etc. By introducing hydrophobic group into polymer 
molecular structure, flocculation performance of the polymer is improved.

Several studies have shown that the polymer structure has a great effect on the flocculation efficiency, such as the $\mathrm{COO}^{-}$group in the anionic polyacrylamide-acrylate copolymer [10], which makes the polymer chain expand by reason of negative charge repulsion between molecular chains. Joo [11] found branched polyamines were more efficient than grafted polyamine during the dye wastewater treatment, because the former had higher charge density than the latter. Khalil [12] prepared six types of anionic starch derivatives and found the type of acidic group, the length of the molecular chain and the chemical formula (grafted or etherified) all had effects on the flocculation performance, which was monitored via estimation of the optimal dosage, transmission and weight removal. Pal [13] reported that the starch with longer $N$-(3-chloro2-hydroxypropyl) trimethyl ammonium chloride (CHPTAC) chains showed the best flocculation performance among the four prepared cationic starches. Despite the studies noted above, there are few previous reports that study the effect of hydrophobic groups in polymer molecules on the flocculation efficiency.

In our opinion, PAM modified with some hydrophobic group must be efficient in wastewater treatment, and be helpful in solid-liquid separation. In this report, three kinds of cationic copolyacrylamide with different hydrophobic alkyl chains were prepared. They were used in the flocculation test and dewatering experiment. Comparisons of these hydrophobically modified PAMs with nonionic PAM and high charged mono-poly(diallyldimethyl ammonium chloride) (PDADMAC) were investigated.

\section{Experimental}

\subsection{Materials}

Sodium hydroxide, acetone, methanol, epichlorohydrin, ammonium persulfate and sodium hydrogen sulfite were analytical grade. Acrylamide, triethylamine, tributylamine and trioctylamine were chemical grade. They were obtained from Shanghai Chemical Reagent Co., Ltd. (Shanghai, China). Kaolin clay was purchased from Guangdong Jieshan chemical plant (Guangdong, China) and was chemical grade. Non-ionic polyacrylamide (PAM) was prepared in the laboratory. The intrinsic viscosity $([\eta])$ was $126.7 \mathrm{~mL} / \mathrm{g}$. Mono-polymer of diallyldimethyl ammonium chloride (PDADMAC) was prepared. The intrinsic viscosity was $86.6 \mathrm{~mL} / \mathrm{g}$ and the cationic charge density was $100 \%$. Deionized water was used in the whole experimental process.

\subsection{Methods}

\subsubsection{Preparation}

The synthesis method described by $\mathrm{Lu}$ [14] was used in the preparation of the hydrophobic modified copolymers. The polymers were synthesized in three steps. First of all, quaternary ammonium salt was prepared with epichlorohydrin and different tertiary amine in acetone at $50{ }^{\circ} \mathrm{C}$ for $36-48 \mathrm{~h}$. After the products were shelved for several days, different crystals were obtained (white granular crystal when the tertiary amine was triethylamine, colorless lamellar crystal when the tertiary amine was tributylamine and colorless acicular crystal when the tertiary amine was trioctylamine.) Secondly, cationic hydrophobic monomers were synthesized with these crystals and acrylamide at the same mole ratio. The reaction lasted $12 \mathrm{~h}$ at $60-70{ }^{\circ} \mathrm{C}$. Thirdly, polymers with different charge density were obtained by changing mole ratio of cationic monomer from $20 \%$ to $100 \%$. They were synthesized at $60-70{ }^{\circ} \mathrm{C}$ for $18-24 \mathrm{~h}$ in nitrogen atmosphere. Ammonium persulfate and sodium hydrogen sulfite were used as initiator. These polymer aqueous solutions were precipitated and extracted with methanol and then dried in a vacuum oven at $60^{\circ} \mathrm{C}$ for $3 \mathrm{~h}$. The yield of the polymerization was calculated with the following equation:

Yield $(\%)=\frac{\text { weight of recovered polymer }}{\text { weight of total monomers }} \times 100 \%$

\subsubsection{Charge density (CD) measurements}

Polymer water solution was titrated with $0.0500 \mathrm{~mol} / \mathrm{L}$ silver nitrate using potassium chromate as indicator. The charge density was evaluated from the following equations:

$$
\begin{aligned}
& \mathrm{CD}(\%)=\frac{n_{\mathrm{c}}}{n_{\mathrm{c}}+n_{\mathrm{AM}}} \times 100 \% \\
& \mathrm{CD}(\%)=\frac{C V}{\left[(m-M C V) / M_{\mathrm{AM}}+C V\right]} \times 100 \%
\end{aligned}
$$

In Eq. (2), $n_{\mathrm{c}}$ is the mole of cationic monomer, which is equals to the used silver nitrate; $n_{\mathrm{AM}}$ is the mole of acrylamide. 
In Eq. (3), $C$ is the concentration of silver nitrate; $V$ is the volume of silver nitrate which is used during the titration; $\mathrm{m}$ is the weight of the polymer; $M$ is the molecular weight of the cationic monomer; $M_{\mathrm{AM}}$ is the molecular weight of acrylamide.

\subsubsection{Intrinsic viscosity $([\eta])$ measurements}

The intrinsic viscosity was determined by an Ubbelohde viscometer at $30 \pm 0.02{ }^{\circ} \mathrm{C}$ in sodium chloride solution $(M=1 \mathrm{~mol} / \mathrm{L})$.

\subsubsection{TEM measurements}

Aqueous solution $(1 \mathrm{~g} / \mathrm{L})$ of each kind of polymer was analysed by $\mathrm{H}-500$ transmission electron microscope (Hitachi, Ltd., Japan) to get the micrograph.

\subsubsection{Flocculation tests}

The kaolin suspension was prepared by mixing $1.5625 \mathrm{~g}$ kaolin in $5 \mathrm{~L}$ deionized water, which was stirred at $500 \mathrm{rpm}$ for $24 \mathrm{~h}$ (turbidity $=248 \mathrm{NTU}$, $\mathrm{pH}$ 7.5). The flocculation test was carried out using a standard jar apparatus (Hubei Meiyu Instrument Co., Ltd., China). The suspension was put into six 1-L breakers and the flocculants were added in as $1 \mathrm{~g} / \mathrm{L}$ aqueous solution. Immediately after the addition of the flocculant, the suspension was stirred at a fast speed of $200 \mathrm{rpm}$ for $3 \mathrm{~min}$, followed by $100 \mathrm{rpm}$ for $6 \mathrm{~min}$. After the floc was settled down for $2 \mathrm{~h}$, the turbidity of the supernatant liquid was measured with digital turbidity meter ZD-1 (Tianjin Analytical Instrument Company, China).

\subsubsection{Dewatering tests}

Polymer solution of $1 \mathrm{~g} / \mathrm{L}$ was added in a $500 \mathrm{~mL}$ cylinder with kaolin suspension. They were mixed by moving a perforated plunger up and down eight times to ensure the suspension was well dispersed by visual inspection. The settling rate of flocculated suspension was determined by recording the time taken for the 'mud-line' to pass between the $450 \mathrm{~mL}$ and $350 \mathrm{~mL}$ marks [15]. After the suspension was settled down for $24 \mathrm{~h}$, the supernatant liquid was carefully sucked out of the cylinder. Surface and free moisture in flocs was removed by TGL-16C centrifuge (Shanghai Zhisun Instrument Co., Ltd., China) at the speed of $4000 \mathrm{rpm}$ for $3 \mathrm{~min}$. Then the weight of the slurry was recorded. The result was the average value of three repeated tests.

\section{Results}

\subsection{Effect of the monomer feed ratio on the copolymerization}

Three series of copolymers of acrylamide and 3-acrylamido-2-hydroxypropyltrialkylammonium chloride were prepared, in which the alkyl groups were ethyl, butyl and octyl, respectively. Their corresponding English abbreviations are PAAHTE, PAAHTB and PAAHTO. The molecular structures of these three kinds of polymers are listed in Fig. 1. The copolymerization results are summarized in Tables 1-3. From these tables, we can see the intrinsic viscosity and the yield decreased when the charge density of the polymer increased. This behavior can be explained as follows, when the feed ratio of cationic hydrophobic monomer increased, the hydrophobicity of the molecule tends to increase too. This makes the molecule hard to be turned into free radical and be polymerized in an aqueous solution. Therefore, it is important in the preparation to adjust the feed ratio of each monomer to a suitable value in order to get the best balance between charge density and intrinsic viscosity, which, respectively, represent the neutralization ability and crossbridging ability of a flocculant.

Table 3 shows that the sample numbers 5 and 6 have a relatively high charge density and intrinsic viscosity compared with the results obtained with ethyl and butyl, which are shown in Tables 1 and 2. The explanation of this phenomenon can be that, due to the dimensions of the octyl chain, the reaction turns into a micellar polymerization [16] in water. This makes it easier for the polymerization of the cationic monomer. At the same

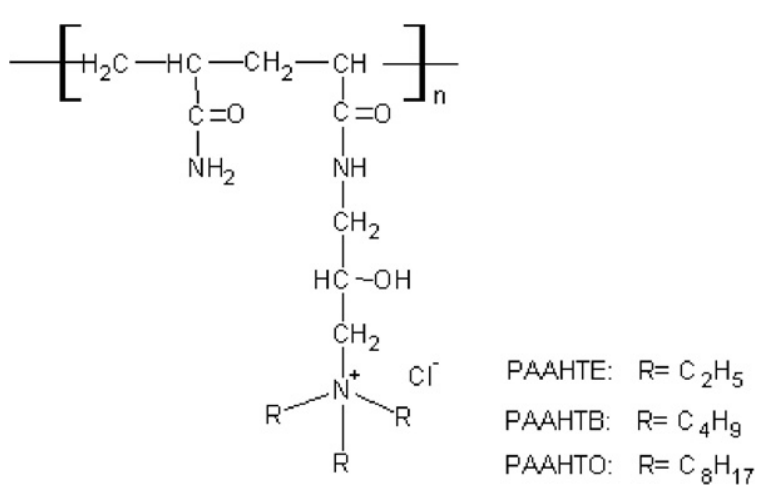

Fig. 1. Molecular structures of three kinds of hydrophobically modified polymers prepared in this paper. 
Table 1

Copolymerization of acrylamide with 3-acrylamido-2-hydroxypropyltriethylammonium chloride

\begin{tabular}{|c|c|c|c|c|c|}
\hline Sample & $\begin{array}{l}\text { Initial hydrophobic } \\
\text { monomer } \\
\text { concentration }\left(\mathrm{mol}^{\circ} \%\right)\end{array}$ & $\begin{array}{l}\text { AM feed ratio } \\
(\mathrm{mol} \%)\end{array}$ & Yield $(\%)$ & $\begin{array}{l}\text { Charge density } \\
(\mathrm{mol} \%)\end{array}$ & {$[\eta](\mathrm{mL} / \mathrm{g})$} \\
\hline 1 & 20 & 80 & 19.77 & 13.11 & 121.8 \\
\hline 2 & 33 & 67 & 26.42 & 19.82 & 88.5 \\
\hline 3 & 45 & 55 & 12.62 & 34.21 & 70.8 \\
\hline 4 & 60 & 40 & 4.70 & 66.00 & 51.0 \\
\hline 5 & 75 & 25 & - & - & - \\
\hline 6 & 100 & 0 & - & - & - \\
\hline
\end{tabular}

Table 2

Copolymerization of acrylamide with 3-acrylamido-2-hydroxypropyltributylammonium chloride

\begin{tabular}{lllrlr}
\hline Sample & $\begin{array}{l}\text { Initial hydrophobic monomer concentration } \\
(\mathrm{mol} \%)\end{array}$ & $\begin{array}{l}\text { AM feed ratio } \\
(\mathrm{mol} \%)\end{array}$ & Yield $(\%)$ & $\begin{array}{l}\text { Charge density } \\
(\mathrm{mol} \%)\end{array}$ & {$[\eta](\mathrm{mL} / \mathrm{g})$} \\
\hline 1 & 20 & 80 & 32.90 & 11.59 & 288.0 \\
2 & 33 & 67 & 39.60 & 16.30 & 184.0 \\
3 & 45 & 55 & 36.10 & 29.80 & -3 \\
4 & 60 & 40 & 2.25 & 32.00 & - \\
5 & 75 & 25 & 1.42 & - & - \\
6 & 100 & 0 & 1.87 & - & \\
\hline
\end{tabular}

Table 3

Copolymerization of acrylamide with 3-acrylamido-2-hydroxypropyltrioctylammonium chloride

\begin{tabular}{|c|c|c|c|c|c|}
\hline Sample & $\begin{array}{l}\text { Initial hydrophobic monomer concentration } \\
(\mathrm{mol} \%)\end{array}$ & $\begin{array}{l}\text { AM feed ratio } \\
(\mathrm{mol} \%)\end{array}$ & Yield (\%) & $\begin{array}{l}\text { Charge density } \\
(\mathrm{mol} \%)\end{array}$ & {$[\eta](\mathrm{mL} / \mathrm{g})$} \\
\hline 1 & 20 & 80 & 34.56 & 27.15 & 305.0 \\
\hline 2 & 33 & 67 & 21.67 & 42.00 & 197.0 \\
\hline 3 & 45 & 55 & 12.14 & 25.80 & 299.0 \\
\hline 4 & 60 & 40 & 9.92 & 56.00 & 91.0 \\
\hline 5 & 75 & 25 & 15.57 & 17.66 & 120.2 \\
\hline 6 & 100 & 0 & 11.96 & 17.39 & 141.9 \\
\hline
\end{tabular}

time, the octyl chains in polymer molecules crosslink each other in the manner of hydrophobic association [17]. This behavior enhances its intrinsic viscosity.

One sample of each family, with similar intrinsic viscosity $([\eta])$ and charge density $(\mathrm{CD})$, was selected. Their analytical data are as follows: PAAHTE: $[\eta]=121.8 \mathrm{~mL} / \mathrm{g}, \mathrm{CD}=13.11 \%$; PAAHTB: $[\eta]=$ $124.1 \mathrm{~mL} / \mathrm{g}, \mathrm{CD}=14.52 \%$; PAAHTO: $[\eta]=120.2$ $\mathrm{mL} / \mathrm{g}, \mathrm{CD}=17.66 \%$. These three samples were used in the following tests mentioned in this paper.

\subsection{TEM images}

The TEM micrographs of the three kinds of hydrophobic modified polymers are listed in
Fig. 2. The monotonous molecule of PAAHTE is displayed in Fig. 2a. The single chain molecule of PAAHTB is displayed in Fig. 2b. The long and thin branches, large net-like molecular structures of PAAHTO are displayed in Fig. $2 \mathrm{c}$ and d. The image of PAAHTO in the micrograph is more bulky and fluffy than the other two. The micrographs proved that some hydrophobic groups in polymer molecule are helpful to enlarge molecule. This may be resulted from the self-forming behavior of the molecules which is associated in the manner of micelle [7]. At the same time, there is repulsive force among the hydrophobic groups, which prevents the molecular chain bonding together. This probably is the reason that the chains of PAAHTO are more stretching and thinner than that of PAAHTE and PAAHTB. 

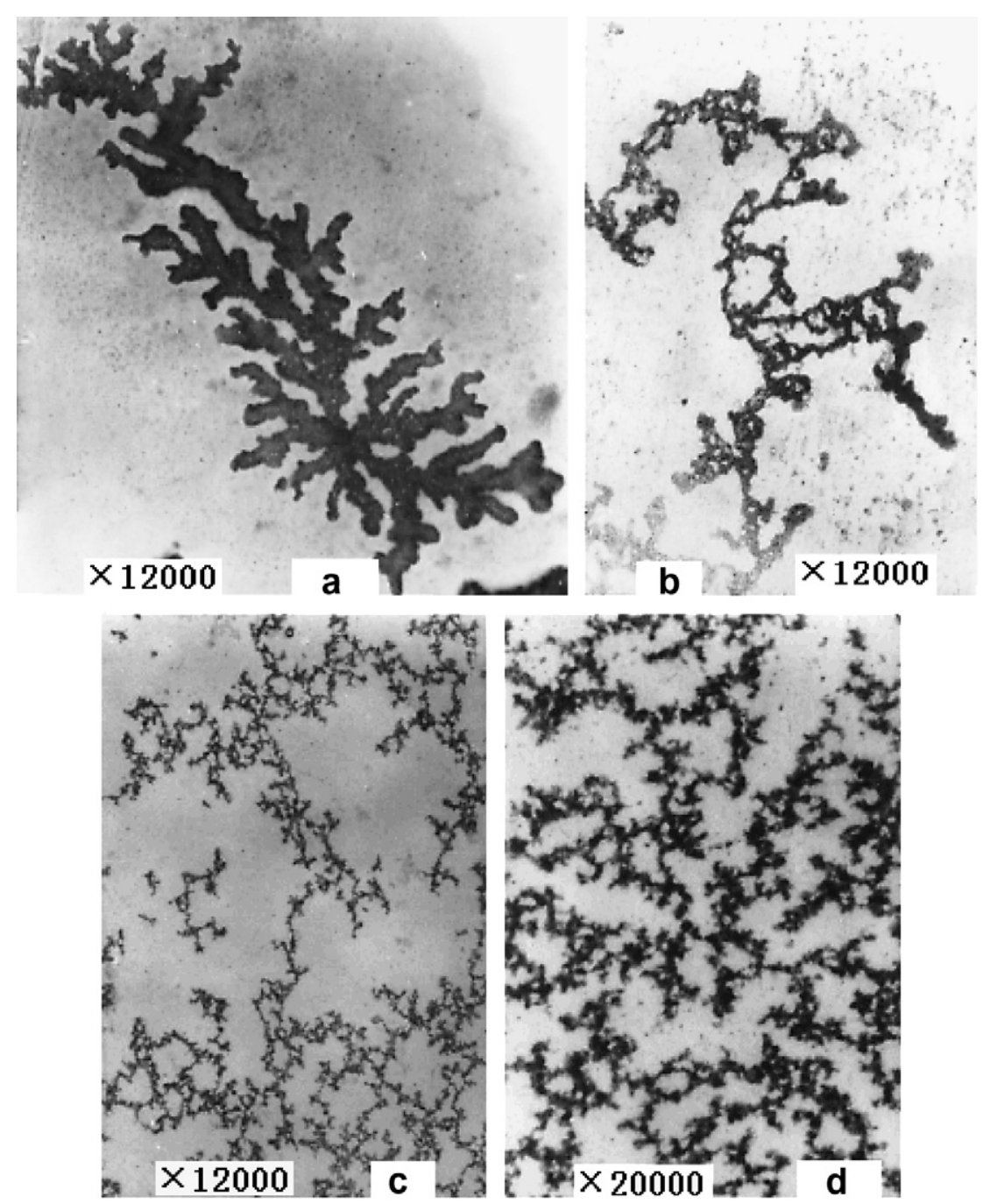

Fig. 2. TEM micrographs of three kinds of hydrophobically modified copolymers prepared in this paper.

\subsection{Effect on turbidity removal}

The performances of the samples in turbidity removal of kaolin suspension were studied. The results are listed in Fig. 3.

With the increase of the dosage, those three samples had similar trend on turbidity removal. The residual turbidity decreased first and then increased. PAAHTO was at a less dosage than PAAHTE and PAAHTB when the best turbidity removal was arrived. From the figure, we can see that PAAHTO is the most effective one in turbidity removal, and PAAHTB is better than PAAHTE. This may be attributed to the long molecular chains of PAAHTO, which make it easier for them to touch each other and get incorporated to form a net

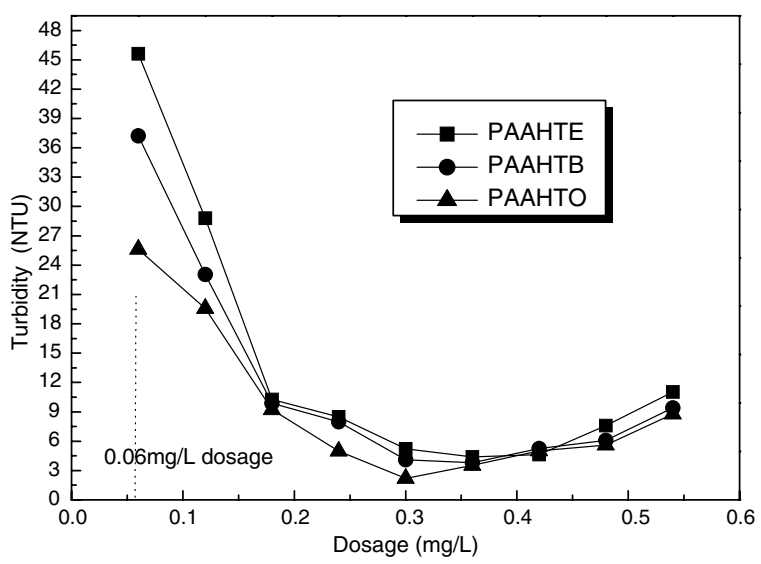

Fig. 3. Turbidity removal of kaolin suspension flocculated by PAAHTE, PAAHTB and PAAHTO. 
structure. At the same time, the long alkyl chain enhances the hydrophobicity of the polymer, and promotes the association among polymer molecules and hydrophobic kaolin particles. As the dosage increased, residual turbidity increased and the deflocculation took place. This is due to the repulsion between the added flocculant molecules and the polymer chains that already adsorbed on the particle surface [13].

Comparison in turbidity removal of PAM, PDADMAC and PAAHTO was investigated. The results in Fig. 4 show that the efficiency of PDADMAC is higher than that of PAAHTO, and this difference is more pronounced at the low dosage of $0.2 \mathrm{mg} / \mathrm{L}$ or lower. The dosage of PAAHTO is about $0.3 \mathrm{mg} / \mathrm{L}$ when its efficiency is the highest. PAM has the lowest efficiency and the highest optimum dosage of $0.5 \mathrm{mg} / \mathrm{L}$. With these phenomena, we can see that the flocculation of the kaolin particles is in the control of neutralization, then the bridge. The neutralization makes the charge of kaolin particles change from negative to neutral, and makes the particle easier to incorporate. PAAHTO has relatively higher charge density than PAM, so it can neutralize part of negative density of kaolin particles. At the same time, hydrophobically modified polymer bridges the suspended kaolin by adsorbing at more than one site on different particles with its long alkyl chains. This behavior enhances the anchor of polymer chain to hydrophobic kaolin particles, promotes the interaction between particles, and affects the flocculation performance.

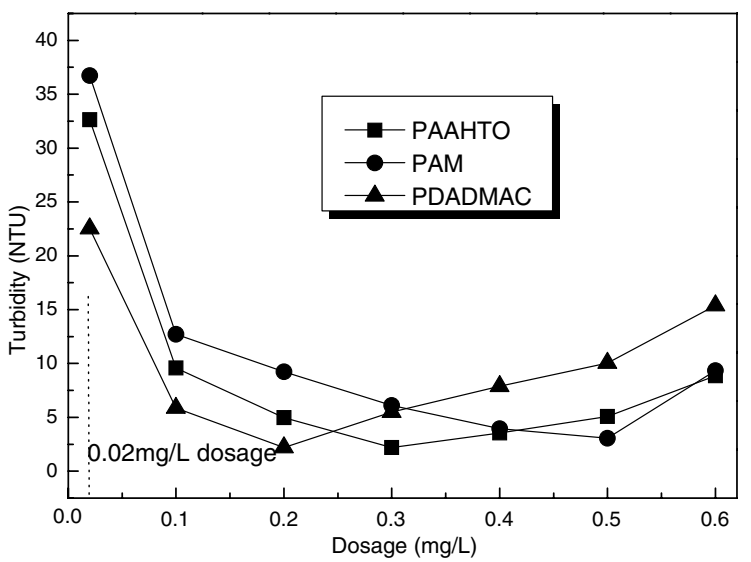

Fig. 4. Turbidity removal of kaolin suspension flocculated by PAAHTO, PAM and PDADMAC.

\subsection{Effect on settling behavior and dewatering efficiency}

The settling performances and dewatering efficiencies of the hydrophobically modified polymers, PAM and PDADMAC are shown in Figs. 5 and 6.

In Fig. 5, only PDADMAC has fast settling rate when the dosage is lower than $0.3 \mathrm{mg} / \mathrm{L}$. This is also attributed to the high cationic density of PDADMAC. Even at low concentration, its neutralization works well in making the negative particles separate from the water as neutral species. But for the hydrophobically modified PAMs and PAM, at relatively low dosage, lacking of junction points, the

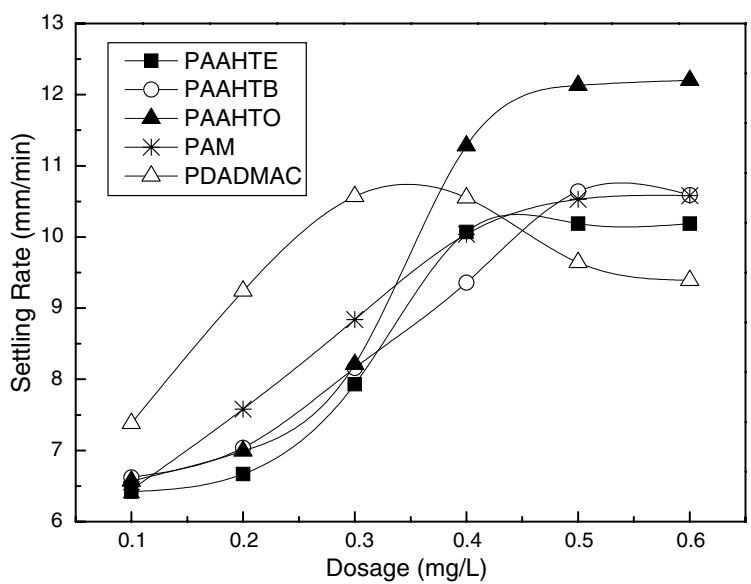

Fig. 5. Settling rate of kaolin suspensions flocculated by PAAHTE, PAAHTB, PAAHTO, PAM and PDADMAC.

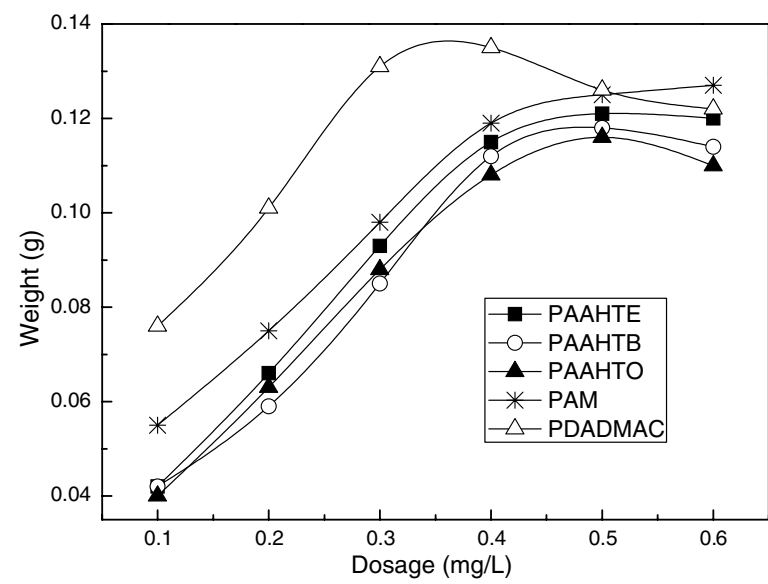

Fig. 6. Sludge weight of kaolin suspension flocculated by PAAHTE, PAAHTB, PAAHTO, PAM and PDADMAC. 
flocs induced by bridging have a lower density than the flocs formed by neutralization. Increasing polymer dosage, more particles are bridged and a significant increase in settling rate is obtained. In the range of $0.3-0.5 \mathrm{mg} / \mathrm{L}$, the settling rate of the hydrophobically modified PAMs increased dramatically, while that of PAM increased gradually. The high settling rate of PAAHTO, $12 \mathrm{~mm} / \mathrm{min}$, was observed when the dosage was higher than $0.45 \mathrm{mg} / \mathrm{L}$, which was much higher than that of PDADMAC and PAM. This may be resulted from the hydrophobic associating behavior between alkyl chains and kaolin particles. There is a process for the molecules chains to anchor on the surface of kaolin particles. Once the critical associating concentration (CAC) [18] is obtained, the high settling rate is obtained.

Dewatering abilities of these five polymers judged with sludge weight are shown in Fig. 6. PDADMAC had the largest sludge weight which could be explained by the poor packing flocs and high retention of inter-floc water in the sediment. With the deflocculation of the kaolin particles, sludge weight decreased at the dosage of $0.4 \mathrm{mg} / \mathrm{L}$. PAM showed better effect on consolidation than PDADMAC, but a little worse than the modified PAMs. As far as the hydrophobically modified polymers, an improvement in dewatering was observed. The polymer with long chains was more effective than that with short chains. Sludge weights decreased slightly when dosage was higher than $0.5 \mathrm{mg} / \mathrm{L}$. These phenomena can be explained by the hydrophobicity of the polymer which results in lower water retention in the sludge. PAAHTB had less weight than PAAHTO at the dosage of $0.15-0.35 \mathrm{mg} / \mathrm{L}$. This may be due to more kaolin particles were flocculated by PAAHTO molecules, which is proved by the turbidity removal in Fig. 3.

\subsection{Effect on sugar refinery wastewater treatment}

The sugar refinery wastewater treatment results are shown in Fig. 7. The three kinds of hydrophobically modified polymers showed good performances, especially PAAHTO. This is attributed to the hydrophobically modified molecular structure. The alkyl groups anchor on the colloid particles in wastewater in a manner of association, and the long chains in polymer molecules bridge each other. Then the colloid particles are coagulated. This behavior makes it easier for flocculant to catch hydrophobic particles. So the longer the alkyl

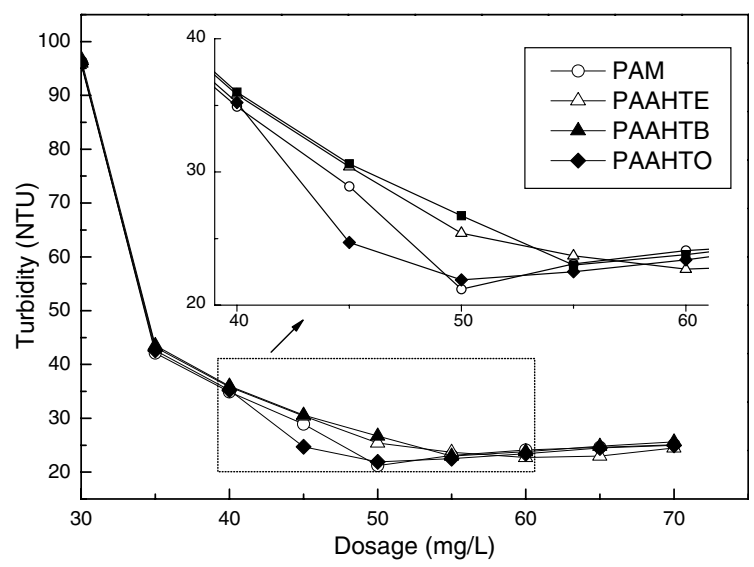

Fig. 7. Turbidity removal of wastewater from sugar refinery flocculated by PAAHTE, PAAHTB, PAAHTO and PAM.

group is, the better the polymer associating ability is. PAM also had high efficiency in the treatment, because it could attach particles by hydrogen bond of the primary amide $\left(\mathrm{CONH}_{2}\right)[10]$ and flocculate the colloid particles by bridging them with its large molecules.

\section{Conclusions}

1. Three series of hydrophobically modified copolymers of acrylamide and 3-acrylamido-2-hydroxypropyltrialkylammonium chloride were prepared and their flocculation performances were studied. In the three copolymer families, PAAHTO, which was modified by octyl chain, had the best performances not only in turbidity removal, but also in the settling behavior and dewatering examination.

2. TEM micrographs showed the hydrophobic group made the molecule stretching and bulky by the self-forming behavior of molecules and the repulsive force among the hydrophobic chains.

3. The flocculation efficiency of hydrophobically modified copolymers, since the point of view of turbidity removal, was higher than the one of unmodified PAM and a little lower than that of PDADMAC. The water retention in sediment that flocculated by the hydrophobically modified copolymers was less than that of PAM and PDADMAC. The settling rate obtained with PAAHTO was the highest one at the concentration of $0.4 \mathrm{mg} / \mathrm{L}$ or higher. 
4. The results presented in this paper show that hydrophobicity of molecule is also an important parameter for flocculant in wastewater treatment.

\section{References}

[1] Tridib Tripathy, R.P. Bhagat, R.P. Singh, Eur. Polym. J. 37 (2001) 125.

[2] J.W. Qian, X.J. Xiang, W.Y. Yang, M. Wang, B.Q. Zheng, Eur. Polym. J. 40 (2004) 1699.

[3] A. Ariffin, Raid S.A. Shatat, A.R. Nik Norulaini, A.K Mohd Omar, Desalination 173 (2005) 201.

[4] A. McFarlane, K. Bremmell, J. Addai-Mensah, Miner. Eng. 18 (2005) 1173.

[5] R.B. Booth, W.F. Linke, US Pat. No. 3147218 (01/09/1964).

[6] D.F. Jacques, B. Mead, J. Bock, US Pat. No. 4734205 (29/ 03/1988)

[7] H.Z. Zhao, Z.K. Luan, B.Y. Gao, Q.Y. Yue, J. Appl. Polym. Sci. 84 (2002) 335
[8] S. Bratskaya, V. Avramenko, S. Schwarz, I. Philippova, Colloids Surf. A 275 (2006) 168.

[9] S. Dragan, A. Maftuleac, I. Dranca, L. Ghimici, T. Lupascu, J. Appl. Polym. Sci. 84 (2002) 871.

[10] P. Mpofu, J. Addai-Mensah, J. Ralston, Miner. Eng. 17 (2004) 411.

[11] D.J. Joo, W.S. Shin, Y.H. Kim, J.H. Kim, J.H. Choi, Sep. Sci. Technol. 38 (2003) 661

[12] Mohamed I. Khalil, Amal A. Aly, Starch-Starke 54 (2002) 132.

[13] S. Pal, D. Mal, R.P. Singh, Carbohyd. Polym. 59 (2005) 417.

[14] L. Lu, G.Q. Wu, Technol. Develop. Chem. Industry (in Chinese) 31 (2002) 8

[15] P. Mpofu, J. Addai-Mensah, J. Ralston, Int. J. Miner. Process. 71 (2003) 247.

[16] Y.J. Feng, L. Billon, B. Grassl, A. Khoukh, J. Francois, Polymer 43 (2002) 2055.

[17] F. Candau, J. Selb, Adv. Colloid Interface 79 (1999) 149.

[18] S.R. Sun, H.J. Zhu, J.H. Luo, Oilfield Chem. (in Chinese) 21 (2004) 173. 